\title{
Grafted hydroxypropyl guargum: Development, characterization and application as flocculating agent
}

\author{
B R NAYAK, D R BISWAL, N C KARMAKAR ${ }^{\dagger}$ and R P SINGH* \\ Materials Science Centre, Indian Institute of Technology, Kharagpur 721 302, India \\ ${ }^{\dagger}$ Department of Mining Engineering, Indian School of Mines, Dhanbad 826 004, India
}

\begin{abstract}
Synthesis of hydroxypropyl guargum-g-polyacrylamide was carried out by ceric ion induced redox polymerization technique at $28 \pm 1^{\circ} \mathrm{C}$. The graft copolymer was characterized by IR and thermal analysis. The flocculation performance of graft copolymer was tested in $1 \mathrm{wt} \%$ coal suspension.
\end{abstract}

Keywords. Graft copolymer; hydroxypropyl guargum-g-polyacrylamide; flocculation; coal suspension.

\section{Introduction}

Recently, polymeric flocculants are extensively being used in the processing of industrial effluent. Polymeric flocculants are convenient to use in comparison to the inorganic coagulating agents. They perform well in small dosage and the flocs formed are of large size and strongly bound to withstand high shear degradation forces.

Among natural and synthetic polymers, synthetic polymers are very effective due to their versatile tailorability, but they have poor shear stability. Natural polymers are of low cost, shear stable, non-toxic and highly biodegradable. Therefore, it has been possible to develop efficient, shear stable and less biodegradable flocculants by grafting synthetic polymers onto the backbone of the natural ones (Swanson et al 1993).

In our laboratory, many graft copolymers have been synthesized by grafting polyacrylamide (PAM) onto starch (Deshmukh et al 1991), guargum (Deshmukh and Singh 1987), xanthangum (Ungeheur et al 1989), amylopectin (Rath and Singh 1997), and sodium alginate (Tripathy et al 1999). It has been observed that graft copolymers are much more efficient than the linear polymers because of their greater 'approachability' to the contaminants, which could be the result of the spreading effect of the dangling grafted PAM chains on the rigid polysaccharide backbone (Singh et al 2000). It was also observed that among all the graft copolymers PAM grafted amylopectin performs the best as a flocculating agent (Rath and Singh 1997; Singh et al 2000) particularly for industrial effluents.

Washing of non-cocking coals for their use in thermal power plant and cement industry is gaining popularity. Several benefits are accrued out of beneficiation of noncocking coals, such as reduction in transportation cost,

*Author for correspondence increased plant utilization factor, and reduction in specific coal consumption (Sinha 1989). It has been observed that PAM grafted hydroxypropyl guargum (HPG) shows better performance than some of the commercially available flocculants in kaolin, iron ore and silica suspensions (Nayak and Singh 2001). In the present study, an attempt has been made to study the flocculation characteristics of HPG- $g$-PAM in coal suspension.

\section{Experimental}

\subsection{Materials}

HPG was procured from Shell, UK. Acrylamide was purchased from E. Merck, Germany. Ceric ammonium nitrate (CAN) was purchased from Loba Chemie, Mumbai. Acetone and hydroquinone were supplied by S.D. Fine Chemicals, India. HPG was used after purification. The coal sample was supplied by Jagannath Colliery, India. The sources of the commercial flocculants along with their chemical nature are given in table 1.

\subsection{Synthesis}

The graft copolymers were synthesized by ceric ion induced redox initiation method in the presence of nitrogen atmosphere. The details of the synthetic parameters are summarized in table 2. In order to remove the homopolymer PAM, the graft copolymers were treated with a mixture of formamide and acetic acid (1:1 by volume) (Fanta 1973).

\subsection{Infrared spectroscopy}

A Perkin-Elmer 630 IR spectrophotometer was used to record the IR spectra within the range of $4000-400 \mathrm{~cm}^{-1}$. The IR spectra were recorded in solid state using a $\mathrm{KBr}$ pellet method. 


\subsection{Thermal analysis}

The thermogravimetric analysis of HPG, PAM, and HPG$g$-PAM 6 was carried out with a Stanton Redcroft (STA $625)$ thermal analyser in nitrogen atmosphere. A heating rate of $10^{\circ} \mathrm{C} / \mathrm{min}$ was employed.

\subsection{Flocculation}

Flocculation tests of coal fine suspension were carried out on column settling method and standard flocculation jar apparatus (supplied by M.B. Instruments, India).

\section{Results and discussion}

In the present investigation, two series of graft copolymers have been synthesized by grafting PAM onto HPG using ceric ion initiator, which initiates free radical sites on the polysaccharides backbone (Hinz and Johnson 1967). In the first series (I-II), the catalyst concentration was varied, while the amount of HPG and PAM were kept constant. In the second series (I and III), the acrylamide concentration was varied at a fixed HPG and catalyst concentration. The average number of grafting sites per backbone molecule depends on the ratio of ceric ion concentration to HPG. Among all the graft copolymers, HPG- $g$-PAM 6, which contains less catalyst concentration and more acrylamide concentration contains fewer number of longer PAM branches. The graft copolymer HPG- $g$-PAM 1 has shorter PAM branches than HPG- $g$ PAM 6, followed by HPG-g-PAM 3. This is reflected in their corresponding intrinsic viscosity values.

The IR spectra of HPG-g-PAM 6 (figure 1), shows a broad absorption band at $3422 \mathrm{~cm}^{-1}$ due to the overlap of $-\mathrm{OH}$ stretching band of hydroxyl group of HPG and $\mathrm{N}-\mathrm{H}$ stretching band of amide group of PAM. Two bands around 1679 and $1642 \mathrm{~cm}^{-1}$ are due to amide-I and amide-II. C-N and C-H stretching bands appear at 1405 and $2928 \mathrm{~cm}^{-1}$, respectively. Another two bands around 1127 and $1182 \mathrm{~cm}^{-1}$ are due to $\mathrm{C}-\mathrm{O}$ stretching frequency of alcohol. $\mathrm{CH}_{2}$ scissoring and $\mathrm{CH}_{2}$ twisting bands appear at 1450 and $1324 \mathrm{~cm}^{-1}$, respectively. $\mathrm{CH}_{2}-\mathrm{O}-\mathrm{CH}<$ stretching bands appear at 1076 and $1026 \mathrm{~cm}^{-1}$. The above mentioned bands are also present in case of HPG and PAM, but at slightly different frequencies (figure 1).

The grafting is also supported by thermal analysis studies. The TGA thermograms of HPG, HPG- $g$-PAM 6, and PAM are shown in figure 2. In case of HPG the weight loss occurs in two distinct zones. The initial weight loss is due to the loss of small amount of moisture in the sample and the second loss is due to the decomposition of polymer. In case of PAM, after the initial loss of weight, there is a continuous loss of weight with increase in temperature up to $240^{\circ} \mathrm{C}$. The degradation after that is due to the loss of $\mathrm{NH}_{2}$ group in the form of ammonia. The degradation of the polymer takes place in two stages in case of HPG-g-PAM 6. The first decomposition is the degradation of both PAM and HPG chains. From $380^{\circ} \mathrm{C}$ to $400^{\circ} \mathrm{C}$, the decomposition of the cyclized product is observed. From the TGA curves, it is obvious that HPG$g$-PAM 6 is more stable as compared to HPG and PAM.

The flocculation performance of the graft copolymers in $5 \mathrm{wt} \%$ coal suspension is depicted in figure 3 . In this figure, the height of the interface between the solid and the liquid is plotted against time. The greater the settling velocity of the floc containing the contaminants, the greater will be its flocculation performance. It is obvious from the results that the performance of HPG- $g$-PAM 6, which contains fewer but longer PAM chains (as evidenced from the intrinsic viscosity values), is better than that of other graft copolymers in coal suspension.

There are two main predominant mechanisms involving polymeric flocculation: electrostatic patch mechanism

Table 1. Sources of the commercial flocculants.

\begin{tabular}{llll}
\hline S1. no. & Name of flocculant & \multicolumn{1}{c}{ Source } & Type \\
\hline 1 & Superfloc N300 & Cyanamid Co., USA & Nonionic \\
2 & Aquaset AS510 & Aquapharm Chemicals Ltd., India & Anionic \\
\hline
\end{tabular}

Table 2. Synthetic details of the graft copolymers.

\begin{tabular}{|c|c|c|c|c|c|c|}
\hline S1. no. & Polymer & $\begin{array}{c}\text { Moles of } \\
\text { MSU }^{\mathrm{a}}\end{array}$ & $\begin{array}{l}\text { Acrylamide } \\
(\mathrm{mol})\end{array}$ & $\begin{array}{l}\text { Amount of } \\
\text { CAN } \\
\left(\operatorname{mol} \times 10^{3}\right)\end{array}$ & $\begin{array}{c}\% \\
\text { Conversion }^{\mathrm{b}}\end{array}$ & $\begin{array}{l}\text { Intrinsic } \\
\text { viscosity } \\
\text { (dl/g) }\end{array}$ \\
\hline I & HPG-g-PAM 1 & $0 \cdot 0018$ & $0 \cdot 14$ & $0 \cdot 10$ & $85 \cdot 38$ & $9 \cdot 82$ \\
\hline II & HPG- $g$-PAM 3 & $0 \cdot 0018$ & $0 \cdot 14$ & $0 \cdot 30$ & $88 \cdot 94$ & $6 \cdot 02$ \\
\hline III & HPG- $g$-PAM 6 & $0 \cdot 0018$ & $0 \cdot 21$ & $0 \cdot 10$ & $83 \cdot 57$ & $10 \cdot 84$ \\
\hline
\end{tabular}



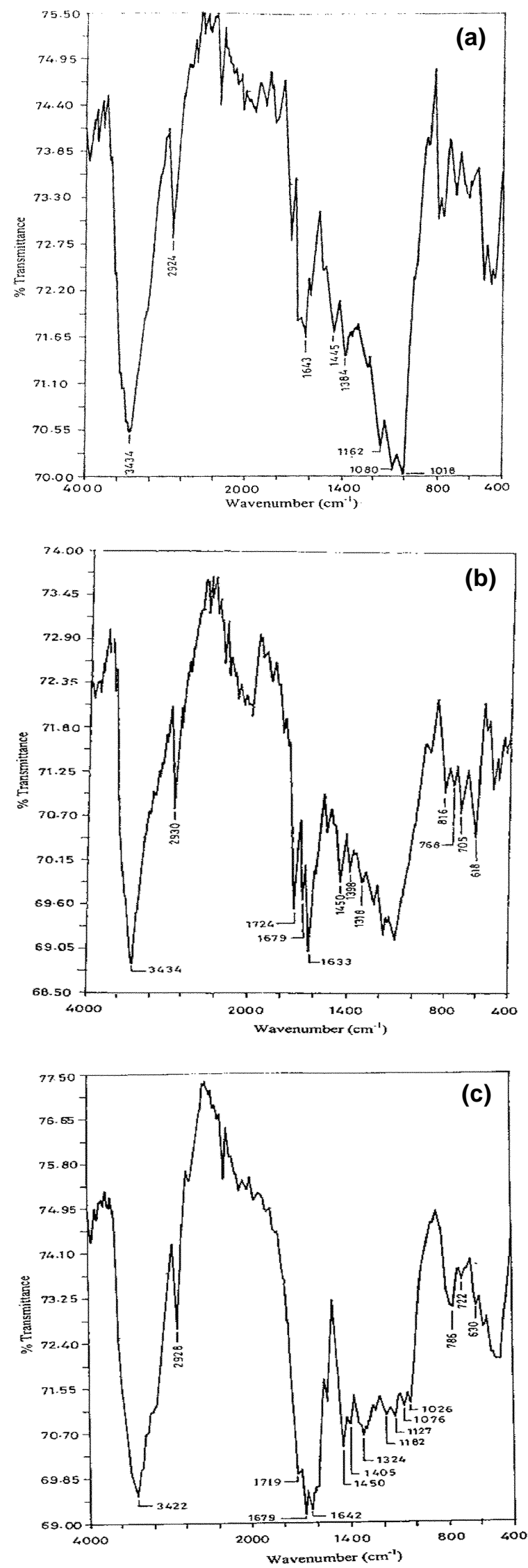

Figure 1. IR spectra of (a) HPG, (b) PAM, and (c) HPG- $g$-PAM 6. and bridging mechanism (Gregory 1996). Here there can be no charge neutralization, so that bridging takes place by adsorption of a polymer molecule at more than one site on a particle or at sites on different particles. When the long chain polymer molecules are adsorbed on the surface of particles, they tend to form loops and extend some distance from the particle surface into the aqueous phase. Their ends also dangle and get adsorbed on the surface of another particle forming a bridge between the particles. The length of the PAM chain should be long for effective bridging, so that they can extend from one

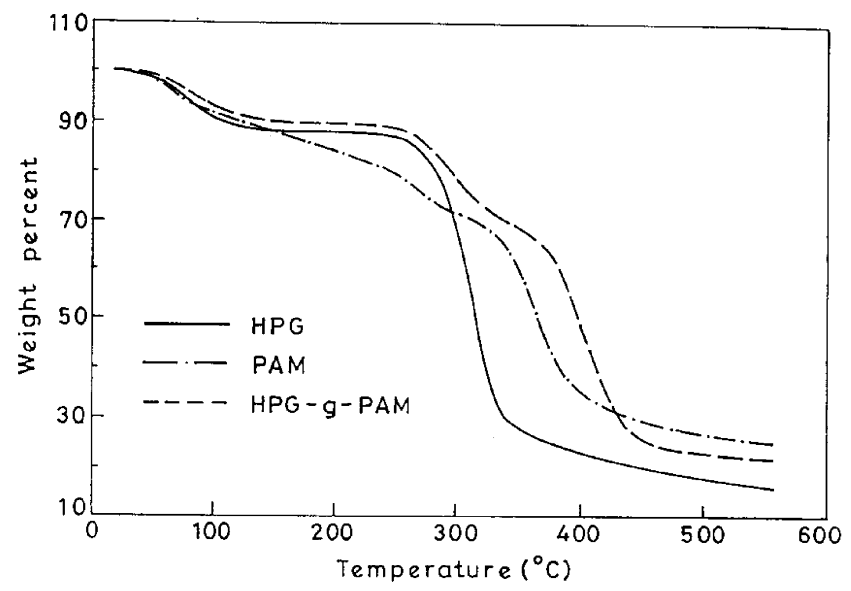

Figure 2. TGA patterns of HPG, PAM, and HPG- $g$-PAM.

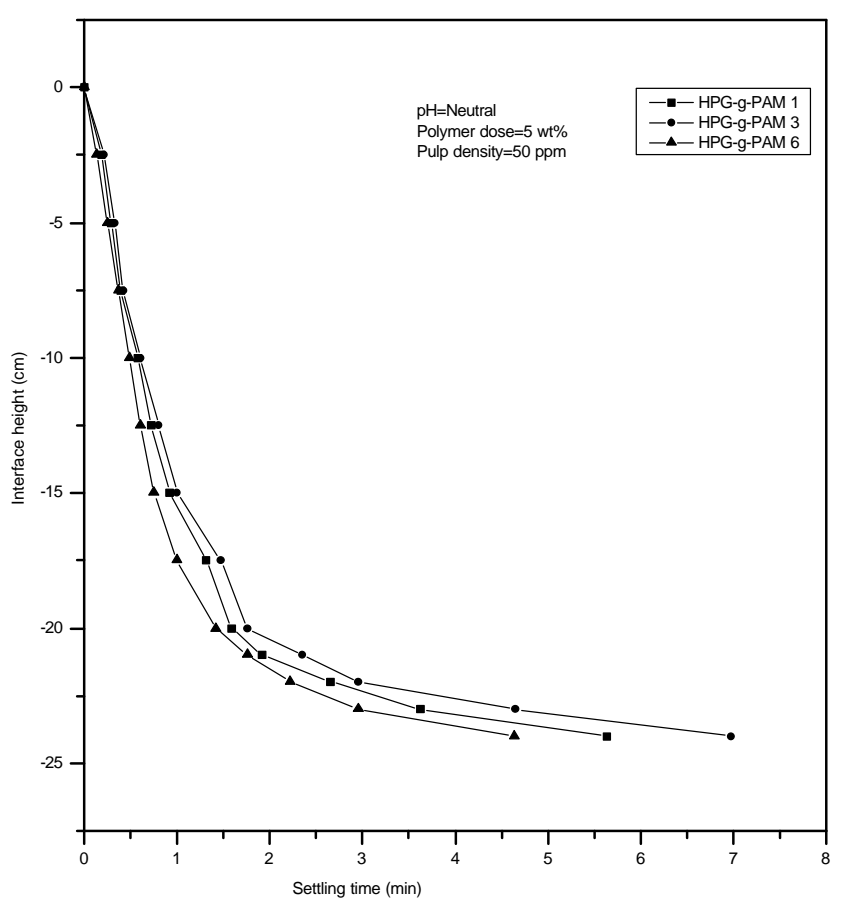

Figure 3. Settling curves for coal suspensions using grafted copolymer. 


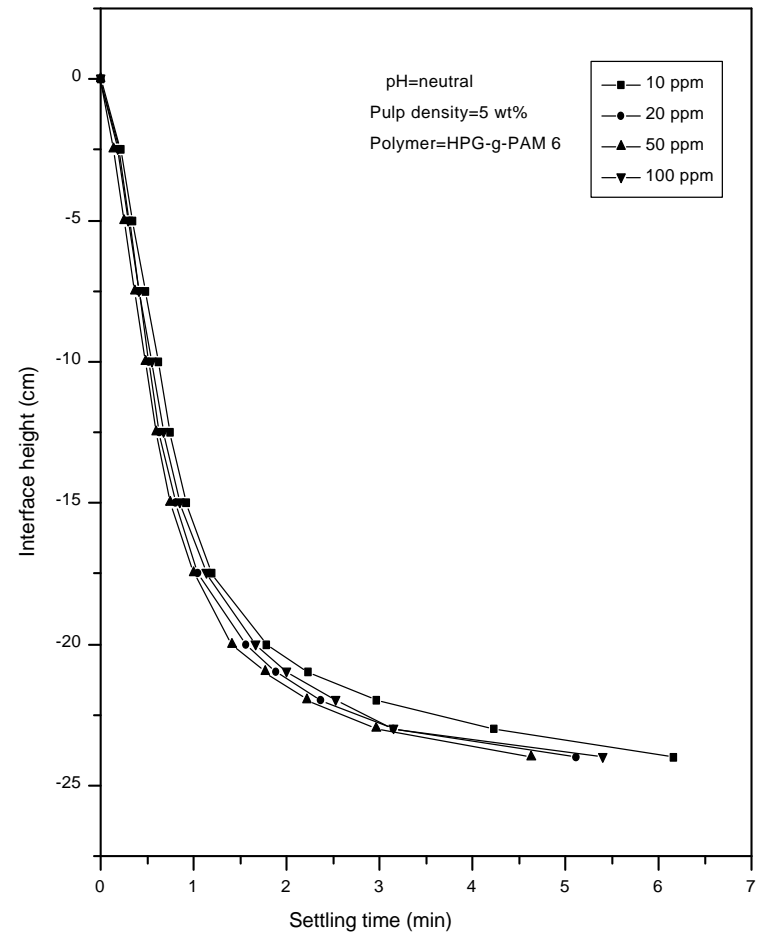

Figure 4. Settling curves for coal suspension using graft copolymer HPG- $g$-PAM 6.

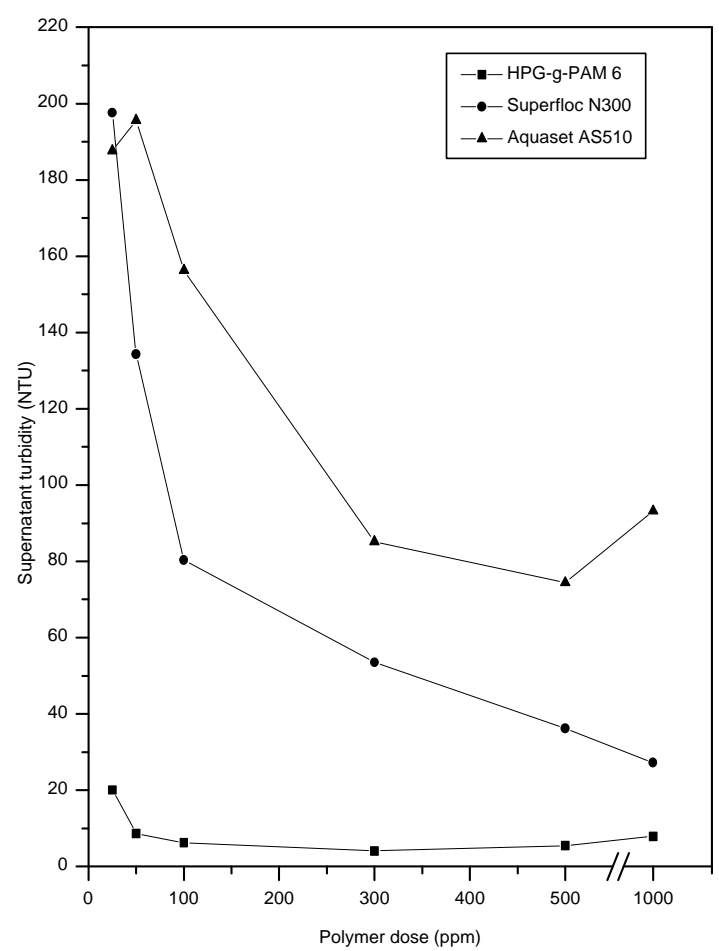

Figure 5. Jar test results of coal suspension (1 wt \%) using commercial flocculants. particle surface to another. Among the graft copolymers, HPG- $g$-PAM 6 has longer PAM chains, it shows better flocculation performance in coal suspension compared to the other. Figure 4 shows that, the optimum polymer dose for HPG- $g$-PAM 6 in coal suspension was found to be $50 \mathrm{ppm}$ (with respect to solid content).

The flocculation performance of the HPG-g-PAM 6 was compared with some of the commercially available flocculants, viz. Superfloc N300 and Aquaset AS510 in $1 \mathrm{wt} \%$ coal suspension. In each case, the turbidity of supernatant liquid after flocculation was plotted against polymer concentration. Figure 5 shows that the graft copolymer still shows better performance than the other commercial flocculants. The reason for better flocculating power of the graft copolymers over the linear polymers is for the effective bridging as already discussed.

\section{Conclusion}

From the above experimental studies, it can be concluded that by grafting PAM chains onto HPG, an effective flocculating agent can be developed for the treatment of coal mines wastewater. Among the grafted product that is having fewer but longer PAM chains (HPG- $g$ PAM 6) performs better than those with shorter PAM chains.

\section{References}

Deshmukh S R and Singh R P 1987 J. Appl. Polym. Sci. 33 1963

Deshmukh S R, Sudhakar K and Singh R P 1991 J. Appl. Polym. Sci. 431091

Fanta G F 1973 Block and graft copolymerization (New York: John Wiley \& Sons) 1 p. 11

Gregory J 1996 Industrial water soluble polymers (UK: Royal Society of Cambridge) p. 62

Hinz H L and Johnson D C 1967 J. Org. Chem. 32556

Nayak B R and Singh R P 2001 J. Appl. Polym. Sci. 81 1776

Rath S K and Singh R P 1997 J. Appl. Polym. Sci. 661721

Singh R P et al 2000 Polym. Eng. Sci. 4046

Sinha P R 1989 J. Mines, Metals and Fuels 34413

Swanson C L, Shogren R L, Fanta G F and Imam S H 1993 J. Environ. Polym. Degrad. 1155

Tripathy T, Pandey S R, Karmakar N C, Bhagat R P and Singh R P 1999 Eur. Polym. J. 352057

Ungeheur S, Bewersdorff H W and Singh R P 1989 J. Appl. Polym. Sci. 372933 